\title{
Knots as a Principle of Form in Modern Art and Architecture*
}

\author{
Dmitri Kozlov \\ Research Institute of Theory and History of Architecture and Urban Planning \\ Moscow, Russia \\ E-mail: kozlov.dmitri@gmail.com
}

\begin{abstract}
The article reviews the origin and development of the theme of knots in the contemporary art and architecture of 20th and 21st centuries. Knots are known from the very origin of the human material culture and for many millennia have become not only convenient and perfect tools and instruments, but also an integral part of traditional arts and crafts. Today, artists, sculptors and architects turn to knots as an effective means of expressiveness and perception of unusual curvilinear forms generated by curved and closed lines in space. Because of their close connection with modern mathematics, knots become one of the leading directions in a new and quick-growing school of mathematical art (math-art). The newest applications of the knot principle in art and architecture include the kinematic form-finding structures proposed by the author, where the knots are used to build transformable surfaces of arbitrary shape.
\end{abstract}

Keywords—knots; art; sculpture; architecture; mathematics; topology; surfaces

\section{INTRODUCTION}

The harmonious relationship between mathematics and art is the most successful in those areas of these both fields of human culture where artistic visual and figurative representations join together with mathematical precision and logical credibility. Both mathematics and art coexist in the human minds and have the creative abilities of man as their common source. In the holistic and harmonious cultures of the past there were no strict and insurmountable boundaries between science and art. Traditionally such adjacent areas of mathematics and art have long been ornamental patterns, symmetry, theory of proportions, various perspective systems, geometry of polygons and polyhedra. In the recent times several new common areas emerged including topology, fractal geometry, and computer graphics.

Knots are known to mankind since the very birth of the human material culture itself. For many millennia knots have become convenient and perfect tools in different areas of

*The Reported study was Funded by Science and Technology Development State Program of the Russian Federation for years 2013-2020 Program of Fundamental Research of State Academies of Science for years 2013-2020, within the Program of Fundamental Researches of Ministry of Construction, Housing and Utilities of the Russian Federation and Russian Academy of Architecture and Construction Sciences; the Research Project 1.6 .3 human practical activities. At the same time they have become an integral part of traditional arts and crafts. The symbolism of knots played an important role in spiritual culture of many peoples of the world as a mean of the visual representation of traditional metaphysical ideas [1]

\section{KNOTS IN SCIENCE}

The study of knots and links is a part of topology that is closely related to algebra, geometry, group theory, matrix theory, number theory and other areas of mathematics. From the point of view of topology, "a knot is a one-dimensional curve situated in ordinary three-dimensional space so that it begins and ends at the same point and does not intersect itself" [2]. Two or more knots can form links, which cannot be divided without breaking of their constituent knots. In fact, every knot is topologically equal or saying in mathematical words homeomorphic to a torus, and its representation in the form of a one-dimensional curve is an abstraction that does not take into account the thickness of the real material object.

The mathematical theory of knots emerged in the second half of the 19th century under influence of the physical hypothesis of that time, according to which the knotted ether vortexes of different shape form the atoms of chemical elements [3]. Nonetheless this hypothesis was proved to be erroneous, in the 20th century the theory of knots found a variety of practical applications in the natural sciences such as physics, chemistry, and biology [ibid]. Now knots and links are considered in science as a form of self-organization in animate and inanimate nature.

Scientific approach to knots as to mathematical objects with great potential of forming stimulated a new aesthetic interest to them in modern art, architecture, sculpture, computer graphics, and decorative design. The theme of knots came into fashion [ibid] and began a part of modern cultural landscape.

\section{THE RISE OF KNOT THEME IN MODERN ART}

Apparently, the first modern artist, appreciated the potential aesthetic content of knots as well as other objects of topology, was Albert Flocon, a French artist and etcher of German origin, "who has both written and drawn on the subject of knots with the imagination of an artist fascinated with topology" [4]. Before depicting knots as an engraving 
in plane, Flocon modeled them from paper in 3D space, resulting in a whole collection of paper models of different knots. It can be assumed that Flocon's enthusiasm with knot modeling was influenced by his studies at the Bauhaus under the guidance of Joseph Albers, who developed a special paper modeling course for art and architecture students.

Mathematically inspirited works of Flocon had a great influence on the creative work of Maurits Cornelis Escher. Escher, having got acquainted with the engravings of Flocon, also built cardboard knot models, which became prototypes of his own famous engravings of knots. In turn, Flocon enthusiastically spoke about Escher's works, speaking of his "amazing discoveries, when it turns out that such seemingly unshakable concepts as up and down, close and far, right and left, can change places" [5].

Flocon and Escher depict knots with the connected ends that are as topological objects, which cannot be untied without breaking them. For these artists, knots are not sacred symbols or elements of decorative patterns that is typical for traditional art. Flocon and Escher base their works on the modern aesthetics of the image of a curved space subjected to external bending forces. The artists offer the viewer to admire the complex continuous forms that look in different ways from different angles of view. Knots become metaphors of complicated play of the inner energy contained in them and affect the sensations of the viewer in an unexpected and unusual way. According to philosopher Gaston Bachelard, a friend and co-author of Flocon, "the twisted and knotted strands, with arcs of tension and spirals of release, are the perfect instruments for a study on the connection of conscience" [4].

\section{R.B. FULLER ON KNOTS}

Interpretation of knotted forms as an external result and perceptually-based representation of the inner and hidden acting forces belongs to Richard Buckminster Fuller - the worldwide known visionary in design and architecture and inventor of modern methods in structural morphology. Fuller included several paragraphs to the structural morphology of knots to his program book "Synergetics", where he formulated the theoretical foundations of forming and structural principles, which he applied in his design and engineering practice. He regarded the knot principle as a manifestation of the pattern integrity and proceeded from the physical treatment of knots similar in its essence to the views of William Thomson (Lord Kelvin), who in the middle of the 19th century put forward the hypothesis that the structures of atoms resemble closed knots [3].

According to Fuller, "each of the chemical elements is a uniquely complex pattern of energy event interrelatednesses which interact inter-interferingly to continually relocalize the involved quantity of energy. These self-interference patterns of atomic element components are in many ways similar to the family of knots that are tied with rope by sailors ..." [6]. By Fuller, the energy concentrating in the knot is generated by a tensile force applied to the free ends of the knot. He writes: "the two ends of the rope immediately outside the knot are pulled away from one another by forces external to the knot, and thus all the attractive forces of Universe operating upon the atoms may result precession ally in keeping the atomic knots pulled together" [ibid].

Speaking of knots as a synergetic phenomenon, Fuller first of all separates the knot as a forming principle from its physical embodiment, such as the material from which the rope is made, its color and texture. Fuller indicates that knots can move freely along the rope in the form of a locally repeating structural configuration, without exerting any influence on its material properties. This observation allowed Fuller to give the next definition of the knot: "the knot is not the rope; it is a weightless, mathematical, geometric, metaphysically conceptual, pattern integrity tied momentarily into the rope by the knot-conceiving, weightless mind of the human conceiver - knot-former" [ibid]. Thus, Fuller moves from the physical to the mathematical interpretation of the knot, to the knot as a structural principle of the intellectual order.

And the rope itself considered in its essence, according to Fuller is a wave physical principle: "What we call the rope itself turns out to be wave phenomena. The fibers themselves were humanly twisted into a spiral wave phenomenon. We are beginning to discover that there is not too much difference between the tactile superficiality of apprehension and the real frequency phenomena that we cannot see in the intervals between the waves" [ibid]. For Fuller, a knot is, above all, a metaphor for complex processes in time, instantaneously "frozen" in space and become visible and tangible. Therefore, Fuller insists on the metaphysical nature of knot and on the principled impossibility of its reduction to any material embodiment. He refers to manifestations and embodiments of the knot principle different physical processes in time, such as flows, currents, waves, and cycles, including human beings: "The metabolic flow that passes through a man is not the man. He is an abstract pattern integrity that is sustained through all his physical changes and processing, a knot through which pass the swift strands of concurrent ecological cycles - recycling transformations of solar energy" [ibid].

Potentials of the Fuller's theory till now are not sufficiently understood and utilized since they are not so much in practical means of structural morphology and innovative methods of design as in a special point of view on form generation as a complex process reflecting the hierarchy of levels of a consequent material embodiment of primary principles of form, one of which is the knot principle.

\section{KNOTS IN MATHEMATICAL ART}

The process of adaptation of the knot forming principles to the contemporary art and architecture inevitably causes attempts of theoretical comprehension of their aesthetics in the context of many thousand-year period of their application in traditional arts and crafts of different peoples of the world. It is not surprisingly that such attempts of analysis of the aesthetic properties of knots in modern and traditional art are made primarily by mathematicians who are interested in art or are involved in artistic creative work. For mathematicians, the aesthetic and artistic properties of knots most often seem 
to be just one of the practical applications of knot theory, even in cases where knots or their images were created long before the mathematical knot theory itself, or when they are created by modern artists working in the traditional paradigms.

The Serbian mathematicians Slavik Jablan and Radmila Sazdanovic, analyze the knots and links as symmetric and mirror curves, the properties of their invertibility from the point of view of knot theory and mathematical art (math-art). Because symmetry is both an artistic and a scientific category, representing itself an intersection of mathematics and art, it is not accidental that the aesthetic comprehension of knots and links begins with their symmetrical examples that is typical not only for modern researchers, but also for people of antiquity, who preferred to use in their woven ornamental patterns only a few types of symmetrical knots [7].

Jablan also considers the aesthetic properties of knots as the manifestation of the symmetry and regularly of their planar projections known as knot diagrams. The diagrams that can be represented as mirror curves traditionally served as modular elements for ornamental patterns and mosaics composed of a limited number of basic modules. In addition to many geometric examples of planar modular patterns combining art and mathematics, the author add the topological ones as "different knot projections occurring in knotwork designs (Islamic, Celtic...), derived from the regular and uniform plane tessellations by using few basic elements 'Knot Tiles"' [8]. Thus, from the mathematical point of view, the "beauty of knots" is equal to the symmetry and modularity of their diagrams - planar projections and to their combinatorial potential.

Jablan notes that "in various fields of (discrete) mathematics, the search for modularity is the recognition of sets of basic elements, construction rules and the exhaustive derivation of different generated structures" [ibid]. He indicates that "modularity is a manifestation of the universal principle of economy in nature: the possibility for diversity and variability of structures, resulting from some (finite and very restricted) set of basic elements by their recombination" [ibid]. Symmetry acts as the basis of combinatorial-modular forming, since "in many cases, the derivation of discrete modular structures is based on symmetry. Using the theory of symmetry and its generalizations (simple and multiple antisymmetry, colored symmetry...) for certain structures, it is possible to define exhaustive derivation algorithms and even to obtain some combinatorial formula for their enumeration" [ibid].

Some of the simplest knots and links have attracted the attention of artists and sculptors since ancient times. These include, in particular, the link of three rings (three trivial knots), and known as the "Borromean rings". This link is characterized by the fact that every pair of two rings in it is unconnected, but all three rings cannot be disconnected without breaking any of them. Since, according to the laws of topology, a ring can be transformed into a triangle, and then Borromean rings can be transformed into entangled Borromean triangles. Jablan writes, that "a hollow triangle is the planar region bounded by two homothetic and concentric equilateral triangles - flat triangular rings. The Australian sculptor Robinson assembled three such triangular rings to form a structure (sculpture entitled 'Intuition'), topologically equivalent to Borromean rings" [ibid].

In modern art, knots are most often depicted in the form of static sculptural compositions or in the form of their planar projections - diagrams, that is, in fact, as woven ornamental patterns. For prototypes of three-dimensional objects, the modern sculptors usually select only the simplest knots and links and perform them with closed ends in the form of an infinite closed line in accordance with their standard mathematical representation.

Modern artists and sculptors turn to knots primarily as to spectacular and sophisticated forms that allow demonstrating the imagery and expression of the closed spatial curves as well as the perception of space itself. In particular, the American mathematician and sculptor Nat Friedman characterizes the beauty of knots as a special aesthetic category, manifested in the construction of closed continuous lines in space and in their perception from different points of view while preserving a holistic multidimensional image. The concept of perception of knotted forms in art Friedman called "hyperseeing" [9].

\section{TRADITIONAL KNOTTING AND MODERN ARCHITECTURE}

The French architect and inventor David Georges Emmerich distinguished two directions in the history of building art. The first was based on structures of heavy massive elements, made, as a rule, of stone. The second was as ancient as the first one and consisted in lightweight structures created from branches and reeds connected with knots of flexible materials. This art was very close to such crafts as basketry and weaving. The first direction has preserved numerous monuments, starting with dolmens, which constitute the main content of the present history of architecture, and from the second direction there is practically nothing left. At the same time the woven building structures of lightweight materials gradually became the source of the origin of the woven decorative ornament in architecture. As Emmerich believed, this is the reason that words such as textiles, textures, and architecture have a common origin [10].

In his argumentation, Emmerich actually follows the architectural theory of Gottfried Semper, which divides architecture into four elements: the ground as the foundation of the building, the wood as its framework, the textiles as wall enclosure and fire as a source of energy to maintain heat in the interior. In his main work Der Stil (1878), Semper gave images of the basic knots used in the building practice, and samples of techniques of weaving and knitting, which look like repeating modules of woven and knotted ornaments. These examples of different types of knotted and woven fabrics served as prototypes of the corresponding textile techniques [11].

The modern Dutch architect Lars Spuybroek, leader of the NOX group, transformed the Semper theory and 
developed his own creative method of architectural shaping, which he named "soft constructivism". Spuybroek regards it as a kind of textile tectonics, in which textiles are transformed into a structural basis of buildings, which forms are based on traditional textile techniques such as weaving, bundling, interlacing, braiding, knitting or knotting. The shaping processes used by Spuybroek are based on complex sequences of scale correspondences that arise in textile tectonics and are implemented by digital and physical modelling. As a result, the microtectonics of the textile surface becomes a macrotectonics of a large building [12].

Spuybroek explains his understanding of the Semper's theory and its relationship to his own textile tectonics. "Semper's concept of textile drives everything, it's the main productive element, the main agent of architectural form. Not at all in the sense of masking, but in the sense of the woven wall being the Urtechnik, the original technique of making architecture. Architectural design is not about having ideas, but about having techniques, techniques that operate on a material level. It's about making matter think and live by itself. A concept that Semper is famous for is the Stoffwechselthese, the transformation of materials, which means buildings aren't made of textile any more, but that textile has been transmaterialised into stone and steel and other constituent parts. So, it's not so much ideas that inhabit matter, but other materials. Textile inhabiting stone. Materials in materials, I find that astonishing. It's an abstract materialism, saving us from idealism and realism at the same time. He was very conscious of that, he wanted to steer in between "speculative aesthetics" and "mere engineering". But it is a very active, evolutionary materialism, a vitalism almost, especially when you remember Stoffwechsel is German for metabolism. Literally it means "change of substance" and even more literally, "change of fabric"!" [13].

\section{KNOTS AS ARCHITECTURAL FORM}

One of the most significant examples of the application of the knot shape in the latest architectural practice is the building of the museum of Mercedes-Benz in Stuttgart was built in 2006 and designed by the Dutch UNStudio founded by architect Ben van Berkel. A distinctive feature of this building is that it was originally designed as a threedimensional spatial object as opposed to the method of flat projections of plans, sections and elevations conventional in architecture. The idea to use the shape of trefoil knot as a prototype of the spatial organization of the Museum became a continuation of the design studies in the field of topology, complex curved surfaces and knots started in UNStudio back in 1996, when it began working on the project of the Central Transport Hub in Arnhem, Germany. In this project, the principle of a surface with a boundary in the form of a knot, known in mathematics as the Seifert surface, was taken for the prototype [14].

The need to isolate various traffic flows from each other led the designers to the idea of "organizing the floor as a landscape, with the entrances to the underground car and bicycle-parking garages organized by bifurcation of the main surface and a main structural element, the so-called "twist". This twist was derived from a spatial interpretation of a figure eight knot" [ibid]. The main building has the form of a minimal surface, combining the functions of optimizing the span of the cover, the organization of pedestrian flows at various levels of the building and ensuring the orientation of passengers. As a result, the architectural appearance of the main building of the Arnhem station became a landmark form of the entire development and one of the city's attractions.

The project statement of the Mercedes-Benz Museum contained a requirement to organize the architectural space of the building in such a way as to provide two independent routes through the expositions - chronological and thematic, with some exhibits of the collection being simultaneously included in both routes. To solve the problem, UNStudio chose the shape of the trefoil knot diagram as a vertical projection and formed the Seifert surface on its basis. Then this surface was translated vertically, so that the same overlap planes were rotated at each of the levels by an angle of 120 degrees relative to each other. Two independent routes for passing through collections of the Museum were organized on the principle of double vertical spirals connecting the respective spaces of the trefoils at each level of the building.

Tobias Wallisser, who designed the Mercedes Benz Museum together with Ben van Berkel, writes believes that "this building became widely known as a technical museum of a new type, as the transformation of movement into an architectural form. It has also become famous as the best example of architecture conceived and designed using digital methods. Spatial organization of the interior produces a memorable impression. The building rotates and turns away from the viewer, at the same time, opening new views into the outer space and the neighboring spaces inside it almost in a kaleidoscopic manner. The spatial composition resembles a baroque space, and the Mercedes-Benz Museum was named as the first building by the new architectural term 'Digital Baroque" [ibid].

\section{RESILIENT KNOTS AND FORM-FINDING OF SURFACES WITH ARBITRARY CURVATURE}

A new emerging direction in modern mathematical art based on the knot principle is the kinetic structures of surfaces formed by complex regular knots and links invented and developing by the author. This direction is a special artistic and scientific interdisciplinary project, with the aim to combine the latest achievements of science and technologies with the modern kinetic art, is also a continuation of experiments with a moving form in avantgarde art of the beginning of the 20th century [15].

This new application of knots and links as mechanical devices for generation of forms was first proposed by the author in as far back as 1985 as a research project of the Laboratory of Architectural Bionics in Moscow [16]. The main function of the knots in these mechanical devices becomes a provision of the structural base for transformable point surfaces and for their regular and reversible spatial transformations. 
Most of the known kinetic structures use the principle of mechanical transformation, assuming an ensemble of geometrically unchangeable elements, connected by hinges. In addition to the mechanical transformation an organic one is also known. It is based on reversible deformation of resilient elements and belongs to characteristic features of the living nature. Such organic nature-like transformable structures can be made as an assembly of continuous, elastic, intertwined rods forming a closed cyclic knot or linkage of several cyclic knots. The energy of resilience arises in the curved elastic rods and retains in them due to the closure of the knot. The same energy is evenly distributed over the continuous structure of the knot due to its cyclicity.

Since the elastic energy in the rods tends to its lowest value, their middle lines tend to coincide with the plane. As a result, all the crossings of the knot become contacting and the set of them forms a mobile point surface, physically given by the latticed structure of the knot. Under the action of external forces applied to the structure of the knot, an excess energy of elasticity arises in it, owing to which the structure tends to leave the plane and assume a spatial position corresponding to its energy minimum. Thus, elastic closed circular knots work as transformable framework structures capable of changing the curvature of the point surface, given by the set of their contacting points [17].

The process of transformation of the structure of an elastic knot is a continuous sequence of changing forms, for example, from a plane to a sphere. This transformation is reversible: the sphere can be rolled up from the plane and reunfolded into it without forming rips or folds on its surface. Changing its shape, the structure of the knot accumulates elastic energy, strains and, thereby, increases its carrying capacity. Any intermediate spatial position of the knot structure can be fixed by additional fastening elements, and thereby the transformed structure can be transformed into a geometrically unchangeable structure.

In the process of transformation of the elastic knot structure, a wave redistribution of the relative location of its point contacts (effect of traveling waves) occurs. As a result of this process the structure changes the areas of its faces, the lengths of the edges and the angles between them, preserving only the invariant of the number of vertex (contacting points) and the connection between them. Owing to these features, the structure of the elastic knot can change its geometry as a whole and form point surfaces of arbitrary Gaussian curvature, namely parabolic, elliptic and hyperbolic and their combinations [18].

In contrast to the generally accepted in the mathematical art approach to knots and links as self-sufficient objects, the aesthetics of which are due to their relatively simple spatial shape, the new application of knots transfers the emphasis of perception to the shapes of surfaces defined by their complex and at the same time regular structures. In this case, the shape of the knots themselves makes a second level of perception, forming a quasi-regular spatial woven lattice.

\section{CONCLUSION}

The impulses of inspiration in the field of form in art and architecture coming from mathematics and natural sciences existed at all times. Today their role as factors affecting the basic principles of form in art and architecture has increased significantly.

Most of the basic principles of form are common to animate and inanimate nature, as well as to human technics, in particular for architecture. The well-known and classic examples of these principles include spiral, branching, radial-ring structure, folds, weaving, minimal surfaces and minimal paths, lattice, modularity, symmetry, etc. All these principles manifest themselves as physical phenomena arising as a result of various natural processes, and also have a mathematical description. Each of these principles corresponds to the structural organization of linear, planar and three-dimensional forms.

Enrichment of the language of primary principles of form in art and architecture is possible due to the inclusion of new and not very popular objects in it, the existence of which also follows from the objective properties of physical space, as well as the simplest geometric figures. One of the most suitable objects for this purpose is a knot, understood as the principle of a three-dimensional form consisted of a single linear closed element embodied in space.

\section{REFERENCES}

[1] A.K. Coomaraswamy. The Iconography of Dürer's "Knots" and Leonardo's "Concatenations", Eye of the Heart, Bendigo, Australia: La Trobe University, 2009, pp. 11-40.

[2] L. Neuwirth. "The Theory of Knots", Scientific American, 140(6), 1979, pp. 84-96.

[3] A.B. Sossinsky. Nœuds, Genèse d'une théorie mathématique, Paris: Seuil, 1999.

[4] J.-M. Kantor. A Tale of Bridges, Nexus Network Journal, 7(2), 2005, URL: https://link.springer.com/content/pdf/10.1007\%2Fs00004-0050020-4.pdf (accessed 20.09.2018)

[5] K.E. Levitin. Geometric Rhapsody, Moscow: Znanie, 1984.

[6] R.B Fuller. Synergetics, New York: Macmillan, 1982.

[7] S. Jablan, R. Sazdanovic. Symmetry and Beauty of Knots, The Proceedings of 7th Conference of the International Society of the Arts Mathematics and Architecture (ISAMA), Valencia, 2008, pp. 155176.

[8] S. Jablan. Symmetry, Ornament and Modularity / Series on Knots and Everything - Vol. 30, Singapore: World Scientific Publishing, 2002.

[9] N.A. Friedman. Hyperseeing, Hypersculptures, Knots, and Minimal Surfaces, URL: https://www.mi.sanu.ac.rs/vismath/nat/index.html (accessed 20.09.2018)

[10] D.G. Emmerich. Structures, Contemporary Architecture (Russian translation of L'architecture d'aujourd'hui), 1, 1969, pp. 4-15.

[11] G. Semper. Style in the Technical and Tectonic Arts, or, Practical Aesthetics, Los Angeles: Getty Research Institute, 2004.

[12] L. Spuybroek. NOX: Machining Architecture, London: Thames \& Hudson, 2004.

[13] M.L. Tramontin. Textile Tectonics: An Interview with Lars Spuybroek, Architectural Design 76(6), 2006, pp. 52-59.

[14] T. Wallisser. Other Geometries in Architecture: Bubbles, Knots and Minimal Surfaces / Mathknow. Mathematics, Applied Sciences and Real Life, Milan: Springer, 2009, pp. 91-111. 
[15] D. Kozlov. Resilient Knots and Links as Form-Finding Structures, The Proceedings of 14th Annual Bridges Conference of Mathematics, Music, Art, Architecture, Culture, Coimbra, Portugal, 2011, pp. 179186

[16] D. Kozlov. Architectural Bionics: From Living Nature to Architecture, The Proceedings of International Conference of Kine[SIS]tem: From Nature to Architectural Matter, Lisbon, Portugal, 2017, pp. 102-110.

[17] D. Kozlov. Knots and Links as Form-Generating Structures / Mathematics and Modern Art, Springer Proceedings in Mathematics, Vol. 18, 2012, pp. 105-115.

[18] D. Kozlov. Structures of Periodical Knots and Links as Geometric Models of Complex Surfaces for Designing, Nexus Network Journal, 15(2), 2013, pp. 241-255. 